\title{
Economic burden of hand, foot, and mouth disease in Vietnam; An evidence for priority setting and efficiency management
}

Bui Thi Nhu Hue ${ }^{1}$, Arthorn Riewpaiboon ${ }^{2 *}$, Luyen Dinh Pham ${ }^{3}$, Thuy Thi Thu Nguyen ${ }^{3}$, Usa Chaikledkaew ${ }^{2}$, Trung Quang $\mathrm{Vo}^{4}$

1 Social, Economic and Administrative Pharmacy (SEAP) Graduate Program, Faculty of Pharmacy, Mahidol University, Bangkok, Thailand

2 Social and Administrative Pharmacy Excellence Research (SAPER) Unit, Department of Pharmacy, Faculty of Pharmacy, Mahidol University, Bangkok, Thailand

${ }^{3}$ Faculty of Pharmacy, University of Medicine and Pharmacy at Ho Chi Minh City, Ho Chi Minh City, Vietnam

4 Department of Economic and Administrative Pharmacy, Faculty of Pharmacy, Pham Ngoc Thach University of Medicine, Ho Chi Minh City, Vietnam

*Corresponding author:

Arthorn Riewpaiboon

arthorn.rie@mahidol.ac.th

Keywords:

Cost of illness; Economic burden; Hand, foot, and mouth; HFMD; Vietnam

\begin{abstract}
Hand, foot, and mouth disease (HFMD) is an acute infection in children caused by enteroviruses. Typical cases of HFMD are mild, but increasingly severe complications involving the central nervous system have been observed. With consideration for this issue, this incidence-based study estimated the total costs (converted to 2017 international dollar rates, Int\$) incurred from pediatric HFMD incidence and treatment in Vietnam. To this end, 306 cases of HFMD in children were analyzed. Most of the patients were 1 to 3 years old (274 cases, 92.9\%), and 234 of the cases (79.3\%) were inpatients. No fatality case was found during the study period. The total cost of illness per episode was Int $\$ 467$, which included direct medical (Int\$228) and direct non-medical (Int\$239) expenditures. The total cost was more than a half of the average monthly wage of Vietnamese individuals.HFMD remains one of the most economically burdensome communicable diseases in Vietnam. Effective solutions to the financial burdens of HFMD should therefore be implemented.
\end{abstract}

\section{INTRODUCTION}

Hand, foot, and mouth disease (HFMD) is an acute enterovirus-induced infection ${ }^{1}$ that most frequenlty afflicts children. Typical cases of HFMD are mild, accompanied with an acute onset of fever; rashes (described typically as maculopapular or papulovesicular rashes) on palms, soles, buttocks, and knees; and vesicle lesions on buccal membranes. However, increasingly severe complications involving the central nervous system have been observed $^{2}$. This is a matter of concern given that HFMD is a common endemic illness all over the world, with more than $50 \%$ of children less than 5 years old potentially vulnerable to enterovirus 71 (EV71) and coxsackievirus A16 infection ${ }^{3,4}$. Until now, no official vaccine or treatment for HFMD has been developed.

Many HFMD outbreaks have been reported globally, especially in the Asia-Pacific region ${ }^{5,6}$, but very limited data have been obtained with respect to the epidemiological characteristics of the disease in the Vietnamese context and other regions. Following a study which was conducted at a Vietnamese central pediatric hospital where 764 children were diagnosed as having HFMD ${ }^{7}$. The majority of these cases were aged up to 5 years, and the diagnosis of all the patients was verified through laboratory confirmation. Among the patients, 51 (29\%) developed severe neurological complications, and three in the group with EV71 infection died. https://www.pharmacy.mahidol.ac.th/journal/ (C) Faculty of Pharmacy, Mahidol University (Thailand) 2021 
In 2007, 2008, and 2009, the total proportions of HFMD cases and lethal conditions due to HFMD in Vietnam were 5,719 cases with 23 deaths, 10,958 cases with 25 deaths, and 10,632 cases with 23 deaths, respectively ${ }^{8}$. Since 2011, the country's Ministry of Health has considered HFMD a severe infectious disease with outbreak potential (class B communicable disease) and has required hospitals to frequently submit official reports regarding the illness. All HFMD data are treated as a component of Vietnam's national communicable disease surveillance system. Until 2015, however, no costing study on HFMD in the country was carried out. Because the official vaccine for the disease is expected to be available in the near future, a cost-of-illness study will be valuable in evaluations of the worth of vaccines and in decision making regarding whether to include selected vaccines in the Expanded Program of Immunization for Vietnam.

Accordingly, this research estimated the total costs incurred from pediatric HFMD incidence and treatment in Vietnam. Our objective was to provide reliable data on HFMD costs to enable the government to manage the disease on setting priority of health problems and conducting studies to explore cost-effective interventions.

\section{MATERIALS AND METHODS}

\subsection{Study design}

This research was an incidence-based cost-of-illness study oriented toward the perspective of society. The study population comprised pediatric patients (up to 15 years old) of the Hospital for Tropical Diseases in Ho Chi Minh City. The inclusion criterion was a primary diagnosis of HFMD that falls under the B08.4 code of the International Classification of Diseases, 10th Revision (ICD-10). The exclusion criteria were (1) incomplete treatment (patient withdrawn from treatment or referred to other hospitals), (2) referral from other hospitals, and (3) the refusal of caregivers to participate in the study or unavailability (contacted via telephone) for a second interview after discharge. Based on time limitation of the study, data collection was conducted in two months. All cases from October to November 2015 were included in the study, so there was no need to calculate sample size.

\subsection{Data collection}

Prospective data were collected through questionnaire-guided interviews with caregivers. The sessions were divided into two stages. The first involved face-to-face interviews conducted on the date at which outpatients visited the hospital and the date at which inpatients were discharged. The second set of interviews was carried out via telephone at day seven after a visit or discharge.

\subsection{Cost calculations}

The costs of illness, from a societal perspective, is composed of direct medical costs, direct non-medical costs and indirect costs. Direct medical costs (DMCs) were defined as costs of the hospital costs including the costs of physician visits, laboratory tests, hospital admission, medical equipment, drugs, and other health facilities. A bottom-up method was applied in calculating the total charges based billing system, then, the ratio of cost to charge (RCC) was employed to adjust the charge data into economic costs. The reference ratio was employed, following a previous hospital cost analysis in Vietnam ${ }^{9}$. DMCs also include patients' family payment for other treatments outside the study hospital.

Direct non-medical cost (DnMC) components were the expenses that the patients' families paid for food, transportation, accommodation, personal facilities, and informal care. Expenditures related to informal care referred to the costs of time lost by caregivers, adjusted on the basis of the reference cost per day, which was calculated as the gross national income (GNI) per capita divided by 365 days. Time loss costs covered only those incurred by caregivers during official work. Such expenses were excluded if caregiving was provided by a housewife, house husband, or an unemployed individual.

Indirect costs were composed of morbidity and mortality costs. As stipulated in the work law of Vietnam, individuals have 42 years of tendering official and legal work, with service beginning at age 18 and ending at age $60^{10}$. Productivity loss was counted in the period of 42 working years. This study also applied a constant 5\% GNI growth rate, which was the average percentage increase in GNI of Vietnam for the last 5 years. The discount rate for succeeding years of this estimation was $3 \%$. 
All original costs were adjusted to the 2017 values indicated in the consumer price index (CPI) of Vietnam for the healthcare industry; the rate applied in this regard was 1.83 (CPI $2015=100$, CPI $2017=183)^{11}$. Finally, these values were converted into 2017 international dollars using the purchasing power parity (PPP) conversion factor ${ }^{12}$, leaving us with a conversion rate of Int $\$ 1=$ VND7,736.

\section{RESULTS}

\subsection{Participant characteristics}

This study involved 306 patients, of whom 295 (96.4\%) fulfilled the criterion for eligibility. Eleven cases(3.6\%) were excluded because clinical characteristics did not correspond with the chosen ICD-10 code. Among the enrolled HFMD cases, those aged 1 to 3 years constituted the majority, accounting for $92.9 \%$ (274 patients) of the sample. Children aged 4 to 5 years old and older made up $5.1 \%$ (15 cases) and $2.0 \%$ (6 cases), respectively. The mean age of the patients was 2 years. Of the sample, 234 cases $(79.3 \%)$ were inpatients, and 61 cases $(21.7 \%)$ were outpatients. In terms of sample composition, the male-to-female ratio was $1.4: 1$.
The HFMD diagnosis of the patients fell under four levels of severity: Grade 1 pertains to uncomplicated cases. Grade 2, which means that the effects of the disease extend to the central nervous system, is divided into Grades $2 \mathrm{a}$ and 2b. Patients with Grades $2 a$ and $2 b$ HFMD receive phenobarbital to suppress myoclonus. Grade 3 indicates nervous system dysregulation, and Grade 4 involves cardiopulmonary failure.

Out of the patients selected, 207 (71.2\%) received prior medical treatment via drug stores (50 cases, 16.9\%), private clinics (94 cases, $31.9 \%$ ), and other hospitals (63 cases, 21.4\%). Only $11.5 \%$ of the patients were administered treatment with the benefit of medical insurance, whereas the expenses of most of the cases (176 patients, 59.7\%) were settled out of pocket by their parents/guardians. The use of insurance during treatment at the study hospital was higher than the usage of the prior treatment, with $38 \%$ (112 cases). The average length of stay of the inpatients was five days, which compelled the caregivers to take a leave from official work for four days. The mean period of time spanning the occurrence of the first symptom to full recovery was twelve days. All the aforementioned data were much lower for the outpatients, who also grappled with a full disease episode length of only seven days (Table 1).

Table 1. Characteristics of the patients.

\begin{tabular}{|c|c|c|c|c|c|}
\hline Characteristics & Number & Percent & Characteristics & Number & Percen \\
\hline Patient age (year & & & \multicolumn{3}{|c|}{ Insurance (used for prior treatment) } \\
\hline $1-3$ & 274 & 92.9 & Out-of-pocket payment & 176 & 59.7 \\
\hline $4-5$ & 15 & 5.1 & Compulsory insurance & 34 & 11.5 \\
\hline$>5$ & 6 & 2.0 & Voluntary insurance & - & - \\
\hline \multicolumn{3}{|c|}{ Mean (SD): 2(1), Median: 1, Range: $1-10$} & Private insurance & - & - \\
\hline \multicolumn{3}{|c|}{ Patient gender } & N/A (no prior treatment) & 85 & 28.8 \\
\hline Male & 174 & 59.0 & \multicolumn{3}{|c|}{ Insurance (used for treatment at the study hospital) } \\
\hline Female & 121 & 41.0 & Out-of-pocket payment & 183 & 62.0 \\
\hline Type of patient & & & Compulsory insurance & 112 & 38.0 \\
\hline IPD & 234 & 79.3 & Voluntary insurance & - & - \\
\hline OPD & 61 & 20.7 & Private insurance & - & - \\
\hline Severity grade & & & \multicolumn{3}{|l|}{ Length of stay } \\
\hline 1 & 68 & 23.1 & \multicolumn{3}{|c|}{ Mean(SD): 5(4), Median: 2, Range: 1-15 } \\
\hline 2 & 226 & 76.6 & \multicolumn{3}{|c|}{ Number of working day loss of caregivers } \\
\hline 3 & 1 & 0.3 & OPD Mean(SD):1(1 & ge: $0-5$ & \\
\hline 4 & - & - & \multicolumn{3}{|c|}{ IPD Mean(SD): 4(4), Median: 3, Range: 0-16 } \\
\hline \multicolumn{3}{|l|}{ Prior treatment } & \multicolumn{3}{|l|}{ Length of episode } \\
\hline Private clinic & 94 & 31.9 & \multirow{5}{*}{$\begin{array}{l}\text { OPD } \\
\text { IPD }\end{array}$} & ge: $3-12$ & \\
\hline Hospital & 63 & 21.4 & & ange: $4-36$ & \\
\hline Drug store & 50 & 16.9 & & & \\
\hline Other & 3 & 1.0 & & & \\
\hline No & 85 & 28.8 & & & \\
\hline
\end{tabular}

\subsection{Direct medical costs}

Data on treatment were divided into three periods: those collected before, during, and after treatment at the study hospital. Significant differences in treatment costs or DMCs 
among the periods were found (Table 2). Almost $70 \%$ of the parents had sought prior treatment at nearby private clinics or drug stores. This initial treatment cost them an estimated average of Int $\$ 23$. The majority of the DMCs were incurred during treatment at the study hospital, amounting to Int $\$ 200$ on average, which was ten times higher than the expenses paid prior to the treatment at the study hospital. After discharge, only some of the patients needed additional medical treatment, for which they paid Int $\$ 5$ as the mean cost. The total DMC for the treatment of one HFMD episode was Int\$228.

The total DMC was calculated mainly on the basis of the DMCs incurred during treatment. Of these costs, the greater proportion was accounted for by the routine service cost of hospitalization (hotel cost), which amounted to Int $\$ 169$ per episode (73.1\%). The other six groups (OPD service, drugs and fluid, lab tests, diagnostic imaging, medical supplies, surgery/procedure) accounted for 36.9\% of expenses, out of which $11.2 \%$ were OPD routine service costs, and $9.1 \%$ were expenses incurred from drug and fluid administration. Check-up costs were recorded only for the outpatients. For the inpatients, the payment for a hospitalization day included the costs of physician visits.

\subsection{Direct non-medical costs and indirect costs}

The total DnMC was estimated at Int $\$ 239$ for a full episode of HFMD. Out of this amount, the largest expense was Int $\$ 112$, which was devoted to food during treatment, but home-prepared meals were not includedin the estimation. Transportation costs were estimated at Int $\$ 59$, and the income loss borne by the parents or caregivers as they spent time taking care of the HFMD patients until recovery was Int $\$ 68$. These individuals did not spend money on accommodations and hiring third-party care providers. The food expenses and income loss accounted for almost 3/4 of the total DnMC. On the basis of the aforementioned figures, the costs of transportation, food, and income loss can be regarded as significant burdens of HFMD.

The patients were children under the working age, and no deaths occurred during the study period. Therefore, no indirect costs were incurred. The total economic burden arising from HFMD comprised all the associated costs of HFMD treatment and direct non-medical costs. Such total was estimated at Int $\$ 467$ per episode.

Table 2. Cost of HFMD.

\begin{tabular}{lcrr}
\hline Cost (2017 Int \$) & Mean (\%) & Median & SD \\
\hline Total DMC & $\mathbf{2 2 8}$ & $\mathbf{1 2 8}$ & 38 \\
DMC-before treatment & 23 & 5 & 29 \\
DMC-after treatment & 5 & 0 & 269 \\
DMC-during treatment & 200 & 100 & 218 \\
$\quad$ Hotel cost & $169(73.1)$ & 91 & 12 \\
OPD service cost & $6(11.2)$ & 0 & 11 \\
Drugs and fluid & $5(9.1)$ & 0 & 13 \\
$\quad$ Lab tests & $8(5.0)$ & 5 & 6 \\
$\quad$ Diagnostic imaging & $2(0.8)$ & 0 & 6 \\
$\quad$ Medical supplies & $2(0.5)$ & 0 & 135 \\
$\quad$ Surgery/procedure & $8(0.3)$ & 0 & $\mathbf{1 8 0}$ \\
\hline Total DnMC & $\mathbf{2 3 9}$ & $\mathbf{2 2 2}$ & 14 \\
DnMC - before treatment & $6(7.6)$ & 2 & 40 \\
DnMC - after treatment & $4(1.0)$ & 0 & 174 \\
DnMC - during treatment & $229(91.4)$ & 217 & 103 \\
$\quad$ Meal & $112(37.3)$ & 106 & 58 \\
$\quad$ Informal care & $58(36.0)$ & 47 & 79 \\
Transportation & $59(26.7)$ & 35 & 0 \\
Hiring & $0(0.0)$ & 0 & 0 \\
\hline Andirect cost & $0(0.0)$ & 0 & $\mathbf{0}$ \\
\hline
\end{tabular}

\section{DISCUSSION AND CONCLUSIONS}

Using an incidence-based approach, this research evaluated the costs of an HFMD episode from disease onset to complete recovery for all new cases admitted over a two-month period at the Hospital for Tropical Diseases in Ho Chi Minh City. We captured all healthcare- 
related costs. Actual resource consumption for patient questionnaires, was analyzed. This study also applied the reference $\mathrm{RCC}^{9}$ for Vietnam hospitals in the calculations.

In this research, costs were recalled by the participants from memory as they were interviewed and retrieved from electronic medical records. The estimations revealed that the average cost per episode was Int $\$ 467$, which was more than $50 \%$ of the 2017 mean monthly wage of Vietnamese individuals (Int\$840) ${ }^{13}$. This cost burden was nearly three times the basic monthly salary levels (Int $\$ 168)^{14}$ of cadres, civil servants, public employees, and armed forces personnel. The costs of HFMD treatment in China ${ }^{15-17}$ and Taiwan ${ }^{18}$ are considerably much higher than those in Vietnam. In this study, the estimated total cost of mild HFMD cases (outpatients) was about US\$34 (US\$1 = VND 22.370) $)^{19}$, whereas those in China and and Taiwan were US\$1860 15 and US $\$ 215^{17}$ (adjusted to 2017 values). This significant difference may be attributed to the higher medical service costs and standards of living in China and Taiwan.

In Vietnam, communicable diseases that affect children other than HFMD impose tremendous burdens on society. A useful approach, therefore, is to compare to these illnesses. Rotavirus infection, for instance, is a major reason for hospitalization and clinic visits among children under 5 years of age, with the disease considerably affecting the use of healthcare resources and the costs incurred from treatment. A study that probed into total costs in societal perspective indicated that rotavirus infection resulted in a cost of US\$174.66 (2014 value $)^{20}$. Converting this cost into VND on the basis of the exchange rate used in that work, adjustments grounded in the CPI, and PPP conversion into 2017 international dollar would yield a rotavirus diarrhea-related expenditure of Int\$891-almost double the HFMD cost (Int\$467).

Several limitations of the study should be considered to grasp the entire picture of the phenomenon of interest. First, our estimation was based on a sample taken from only one tertiary hospital in Ho Chi Minh City, thus presenting difficulties in generalizing the estimations to the entire country. Second, EV71 and related enteroviruses are associated with various clinical symptoms, including respiratory problems, febrile rashes, neurological complications, and gastrointestinal illnesses. Because HFMD is only one of the diseases caused by enteroviruses,
HFMD, as indicated in medical records and actual illness-driven burdens determined through an analysis of the cost-effectiveness of vaccination must be higher than the values derived in this work. Third, the definition of informal care costs adopted in this research could cause an underestimation of HFMD expenditures as we did not consider the opportunity costs of caregivers such as housewives, house husbands, and other individuals engaged in unofficial work. Finally, no Grade 4 cases or death due to HFMD were observed during the study period. We recommend that future cost-of-illness studies on HFMD adopt a longer time horizon spanning an entire year to capture all situations.

In conclusion, HFMD remains one of the most alarming diseases that escalate to annual outbreaks in Vietnam. EV71-associated diseases, particularly HFMD, have caused serious problems for patients and their parents as well as Vietnam's healthcare system and society. The development of a vaccine, which is considered the most effective preventive measure against disease, is currently ongoing.

On the basis of societal perspectives, this research comprehensively examined all opportunity costs arising from HFMD. This is the first research in Vietnam to inquire into the financial burdens of HFMD on the basis of data from medical records and interviews with caregivers of nearly 300 children. This work is also the first to provide evidence-based nationally representative data on the economic losses stemming from HFMD. The results derived in this study can serve as guidance in health problem priority setting and essential data for further economic evaluations of HFMD prevention and control interventions, such as vaccine programs, in the near future. Overall, the findings illustrated that the necessary and effective solutions to the considerable hardships imposed by HFMD on society are incidence management and severity control.

\section{ACKNOWLEDGEMENTS}

This study was a part of the Master of Science Program on Social, Economic and Administrative Pharmacy. The research grant was supported by the program. We also would like to acknowledge with sincere thanks Ms. Vo Thi Ngoc Hue, Dr. Nguyen Minh Quang, Dr. Nguyen Thanh Dung, Dr. Nguyen Thi My Thanh, Dr. Phan Tu Quy, Mr. Dang Tran Minh Phuong and colleagues at Pediatric C Department, ICU, Pharmacy Department for helping with data 
collection at the Hospital for Tropical Diseases in Ho Chi Minh.

\section{Conflict of interest}

The authors declare no conflicts of interest arising in this research.

\section{Funding}

None to declare.

\section{Ethical considerations}

This study was approved by the Ethics Review Committee of the Hospital for Tropical Diseases in Ho Chi Minh City (approval number: 08/HĐĐĐ).

\section{Article info:}

Received August 18, 2019

Accepted February 12, 2020

\section{REFERENCES}

1. World Health Organization. A Guide to Clinical Management and Public Health Response for Hand, Foot and Mouth Disease (HFMD): Manila: WHO Regional Office for the Western Pacific; 2011.

2. Ooi MH, Wong SC, Lewthwaite P, Cardosa MJ, Solomon T. Clinical features, diagnosis, and management of enterovirus 71. Lancet. 2010;9(11):1097-105.

3. Zhu Z, Zhu S, Guo X, Wang J, Wang D, Yan $\mathrm{D}$, et al. Retrospective seroepidemiology indicated that human enterovirus 71 and coxsackievirus A16 circulated wildly in central and southern China before large-scale outbreaks from 2008. Virol. J. 2010;7:300.

4. Ji Z, Wang X, Zhang C, Miura T, Sano D, Funamizu $\mathrm{N}$, et al. Occurrence of hand-foot-and-mouth disease pathogens in domestic sewage and secondary effluent in Xi'an, China. Microbes Environ. 2012;27(3):288-92.

5. Schmidt NJ, Lennette EH, Ho HH. An apparently new enterovirus isolated from patients with disease of the central nervous system. J Infect Dis. 1974;129(3):304-9.

6. Solomon T, Lewthwaite P, Perera D, Cardosa MJ, McMinn P, Ooi MH. Virology, epidemiology, pathogenesis, and control of enterovirus 71 . Lancet Infect Dis. 2010;10(11):778-90.

7. Tu PV, Thao NT, Perera D, Huu TK, Tien NT, Thuong TC, et al. Epidemiologic and virologic investigation of hand, foot, and mouth disease, southern Vietnam, 2005. Emerg Infect Dis. 2007;13(11):1733-41.
8. Vietnam Ministry of Health. Health Statistic Yearbook 2011. Hanoi: Medical Publisher; 2012.

9. Riewpaiboon A, Trung V, Usa C, Minh V. Hospital cost analysis in developing countries: A methodological comparison in Vietnam. Asian $\mathbf{J}$ Pharm. 2018;12(01):8-18.

10. Vietnam National Assembly. Vietnam Labor Code No.10/2012/QH13 published on 2012 Jun 18. Hanoi. 2012. Available from: http://vbpl.vn/TW/Pages/vbpqtoanvan.aspx ?ItemID $=27615 \&$ Keyword $=$ luat $\% 201$ ao \%20dong. [cited 2015 Dec 15].

11. General Statistics Office. Consumer Price Index Gold and USD Price Indexes. 2017. Available from: https://www.gso.gov.vn/Modules/Doc_Download.asp $\mathrm{x}$ ?DocID=22608. [cited 2015 Dec 20].

12. World Bank. PPP Conversion Factor, GDP (LCU per International \$). 2015. Available from: https://data.world bank.org/indicator/PA.NUS.PPP. [cited 2015 Dec 20].

13. Dan M. Mean wage of Vietnamese worker was estimated at VND 6,500,000 per month. Vietnam Times of Finance. 2017 Jan 20. Available from: http://thoibaotaichinhvietnam.vn/pages/xahoi/2017-08-22/luong-binh-quan-nguoi-lao-dongnam-2017-uoc-dat-65-trieu-dong-46955.aspx. [cited 2019 Feb 13].

14. Decree on the Statutory Pay Rate for Public Officials, Public Employees and Armed Forces' Personnel. No. 47/2017/ND-CP, Vietnam Government. Hanoi; 2017.

15. Wang ZL, Xia AM, Li YF, Su HL, Zhan LW, Chen YP, et al. Socioeconomic burden of hand, foot and mouth disease in children in Shanghai, China. Epidemiol Infect 2016;144(1):138-43.

16. Gan ZK, Jin H, Li JX, Yao XJ, Zhou Y, Zhang XF, et al. Disease burden of enterovirus 71 in rural central China: A community-based survey. Hum Vaccin Immunother. 2015;11(10):2400-5.

17. Zheng Y, Jit M, Wu JT, Yang J, Leung K, Liao Q, et al. Economic costs and health-related quality of life for hand, foot and mouth disease (HFMD) patients in China. PLoS One. 2017;12(9):e0184266.

18. Liu DP, Wang TA, Huang WT, Chang LY, Wang ET, Cheng $\mathrm{SH}$, et al. Disease burden of enterovirus infection in Taiwan: Implications for vaccination policy. Vaccine. 2016;34(7):974-80.

19. World Bank. Official Exchange Rate (LCU per US\$, Period Average). 2015 Available from: https://data.worldbank.org/ indicator/PA.NUS.FCRF?end $=2017$ \&locations $=$ VN\&start $=1983 \&$ view=chart. [cited 2015 Dec 20].

20. Riewpaiboon A, Shin S, Le TP, Vu DT, Nguyen TH, Alexander N, et al. Cost of rotavirus diarrhea for programmatic evaluation of vaccination in Vietnam. BMC Public Health. 2016;16(1):777. 